\title{
DetecÇÃo de AVARIAS EM Motores AssínCRONOS DE INDUÇÃo
}

\section{RESUMO}

O motor assíncrono de indução é, de facto, a máquina actualmente preferida para a grande maioria dos accionamentos, graças à sua fiabilidade, robustez e baixo custo. Dado que ocupa um lugar preponderante no processo produtivo têm-se desenvolvido diversos métodos de detecção de avarias que permitem diagnosticar qualquer tipo de defeito e quantificar o seu grau de severidade.

\section{INTRODUÇão}

Quando se aborda a temática do diagnóstico de avarias dos motores de indução não podemos deixar de ter presente que a forma de tratar o assunto está intimamente ligada à dimensão da máquina, à sua localização e à função que desempenha no processo em que está inserida.

Assim, como é óbvio, os custos de paragem de um motor de grande dimensão podem justificar a existência dos meios de diagnóstico mais sofisticados, no sentido de evitar a interrupção de serviço.

Por outro lado, motores de menor dimensão podem também desempenhar um papel tal que a sua interrupção pode ter custos elevadíssimos de reinicialização do processo, como por exemplo, no caso de linhas de produção em que o processo inclui accionamentos que estão relacionados com a formação e solidifição da alma condutora dos cabos eléctricos. Se houver uma paragem súbita o metal solidifica ao longo do processo sendo necessário desmontar todo um sistema complexo levando à perda de produção de várias horas ou dias.

A acessibilidade pode ser o factor determinante na estratégia de diagnóstico, como é o caso de ventiladores em condutas, bombas submersíveis ou máquinas em ambientes perigosos.

O método de diagnóstico a implementar depende também do facto da máquina ter parado por avaria ou continuar em funcionamento.

\section{CASO EM QUE A AVARIA PROVOCA PARAGEM DO MOTOR}

Quando a avaria leva à paragem do motor o diagnóstico deve atender às especificidades de acessibilidade e dimensão numa primeira fase.

Não é demais lembrar, que o primeiro passo numa situação de paragem de um motor, deve iniciar não, pela análise do motor, como parece lógico, mas pela análise das grandezas de alimentação do mesmo e do bom estado das protecções eléctricas, fusíveis e relé térmico.

De seguida devem ser levados a cabo testes eléctricos e mecânicos fundamentais:

- Verificação dos valores das resistências dos enrolamentos que deverão apresentar valores semelhantes nas três fases; medição da resistência de isolamento entre enrolamentos e entre os enrolamentos e a carcaça da máquina.

Os resultados destes testes podem dar indicações úteis relativamente à existência de curto-circuitos entre espiras e entre espiras e a massa. Além disso, testa também a possibilidade do circuito eléctrico de um enrolamento estar interrompido.

- No caso do veio do rotor estar acessível, rodá-lo para verificar se existe atrito anormal ou demasiada prisão, analisando-se assim, se o rotor atrita no estator, se a carga oferece demasiado binário resistivo e se os rolamentos estão gripados.

Além dos testes acima descritos, não é de desprezar a verificação da existência de "cheiro a queimado" junto ao motor que pode indiciar um sobreaquecimento da máquina com consequente deteriorização dos isolamentos nomeadamente dos vernizes utilizados. 
A avaria que não obriga à paragem do motor pode manifestar-se de diversas formas como aumento de temperatura, perda de potência, binário ou velocidade oscilantes, aumento de consumo, ruído, vibração, etc..

Nesse caso, os métodos indirectos de diagnóstico podem ser úteis na identificação do tipo de avaria podendo mesmo constituir um meio de acompanhamento da sua evolução controlando o nível de severidade até ser possível uma interrupção programada para reparação.

Para esse efeito, têm sido desenvolvidos vários métodos que recorrem à monitorização de diversas grandezas associadas ao funcionamento do motor, como por exemplo, a intensidade de corrente de alimentação, o fluxo magnético, as vibrações, o ruído, o binário, a velocidade e a temperatura.

Seguidamente serão apresentados alguns métodos de diagnóstico mais utilizados na pesquisa de avarias eléctricas no estator e no rotor, assim como de avarias mecânicas no rotor, nos rolamentos e na carga mecânica acoplada.

\subsection{DiagNóSTICO DE AVARIAS ELÉCTRICAS}

\subsubsection{AVARIAS NO ESTATOR}

A detecção de espiras em curto-circuito nos enrolamentos do estator pode ser feita por análise das correntes de alimentação do motor representadas a duas dimensões a partir da mudança de referencial do sistema trifásico para o sistema de coordenadas $\mathrm{P} Q \mathrm{Q}$ através da transformada de Park.

As componentes do vector de Park $\mathrm{i}_{\mathrm{D}}$ e $\mathrm{i}_{\mathrm{Q}}$ podem ser obtidas das correntes de alimentação $i_{1}, i_{2}$ e $i_{3}$ a partir das seguintes expressões:

$l_{D}=\frac{\sqrt{2}}{\sqrt{3}} \cdot 1_{1}-\frac{1}{\sqrt{6}} \cdot 1_{2}-\frac{1}{\sqrt{6}} \cdot 1_{3}$

$\mathrm{I}_{\mathrm{Q}}=\frac{1}{\sqrt{2}} \cdot 1_{2}-\frac{1}{\sqrt{2}} 1_{\mathrm{a}}$

No caso de um sistema de alimentação ideal com cargas equilibradas, obtêm-se a representação no plano XY de uma figura de Lissajou em forma de circunferência dada pelas componentes do vector de Park simplificadas:

$$
\begin{aligned}
& 1_{D}=\frac{\sqrt{6}}{2} \cdot 1_{\mathrm{N}} \operatorname{sen}(\omega t) \\
& 1_{Q}=\frac{\sqrt{6}}{2} \cdot 1_{M} \operatorname{sen}\left(\omega t-\frac{\pi}{2}\right)
\end{aligned}
$$

Sendo iM o valor máximo da corrente por fase (A), $\omega$ a frequência angular (rad/s) e t a variável tempo (s).

A representação $\mathrm{XY}$ das componentes do Vector de Park permite detectar a existência de espiras em curto-circuito nos enrolamentos do estator (Fig. 1).

Este método de diagnóstico "on-line" baseia-se no aparecimento de uma forma elíptica da representação XY das componentes do Vector de Park da corrente do motor, cujo alongamento elíptico é proporcional ao grau de severidade da avaria e a orientação do eixo maior está associada à fase avariada [1].

É de referir que a representação das componentes do vector de Park da corrente de alimentação do motor sem avaria (Fig. 1 - esquerda), não é uma circunferência perfeita devido à existência de harmónicos na rede.

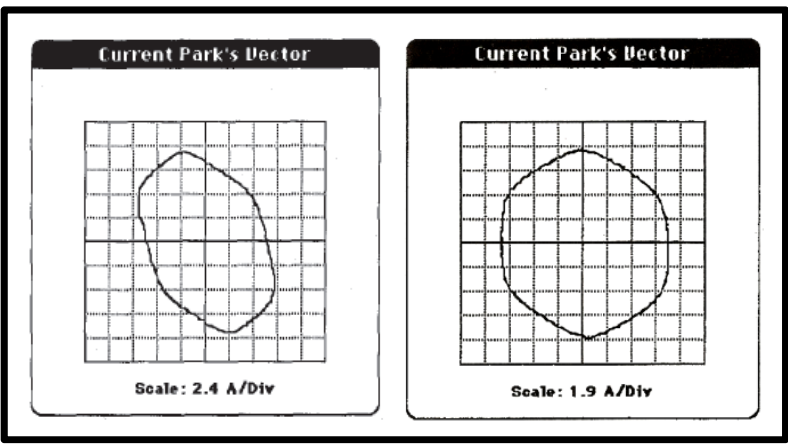

Figura 1- Vector de Park da corrente de alimentação de um motor: sem avaria (esquerda) e com curto-circuito de 18 espiras do estator (direita) [1]

\subsubsection{AVARIAS NO ROTOR}

\subsubsection{MÉTODO DAS COMPONENTES ESPECTRAIS DA CORRENTE}

A detecção de barras do rotor partidas ou fissuradas pode ser feita através da inspecção das componentes espectrais da corrente absorvida pelo motor [2] nas seguintes frequências:

$f_{\mathrm{gs}}=(1 \pm 2 \mathrm{~s}) \mathrm{f}$ 
Sendo $\mathrm{f}_{\mathrm{SB}}$ a frequência $(\mathrm{Hz})$ das bandas laterais resultantes da avaria no rotor, s o deslizamento (\%) e f a frequência da tensão de alimentação $(50 \mathrm{~Hz})$.

Na Figura 2 é fácil identificar o aparecimento de bandas laterais que surgem nas frequências características correspondentes à ruptura de espiras ("barras") do circuito rotórico.

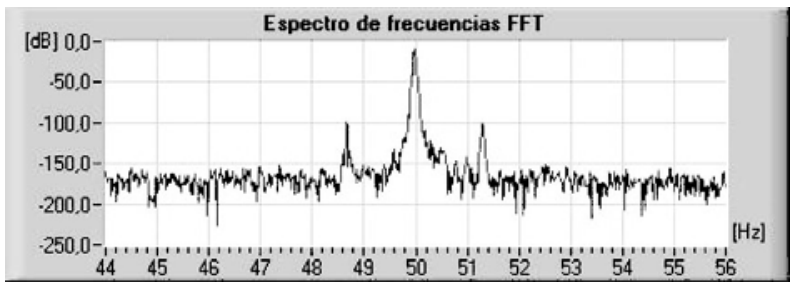

Figura 2 - Espectro de frequências da corrente absorvida pelo motor mostrando claramente a existência de bandas laterais que indiciam uma avaria nas barras do rotor [2]

\subsubsection{MÉTODO DAS COMPONENTES ESPECTRAIS DO FLUXO DE FUGAS}

A aquisição de dados do campo magnético de fugas com vista à análise do seu espectro pode ser feita facilmente colocando sensores de campo magnético no exterior da máquina como mostra a Figura 3.

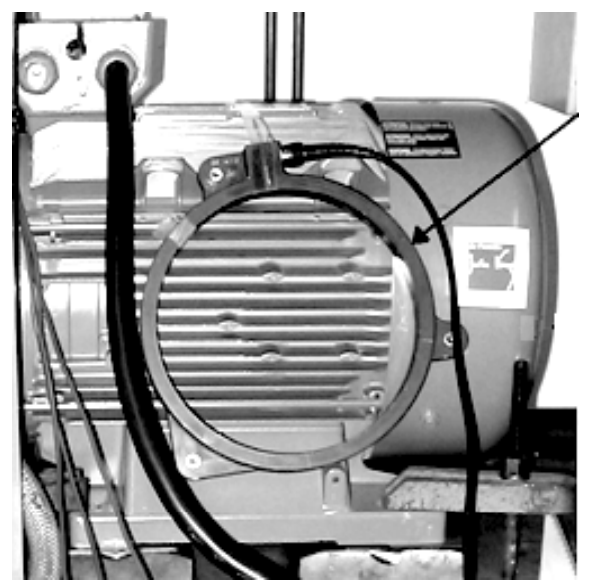

Figura 3 - Detecção de defeito no circuito rotórico através da medição do fluxo magnético: motor com transdutor de fluxo magnético [3]

O defeito devido a barras do rotor defeituosas também pode ser detectado por identificação de determinadas frequências no espectro do campo magnético de fugas medido no exterior da máquina (Figura 4) [3]

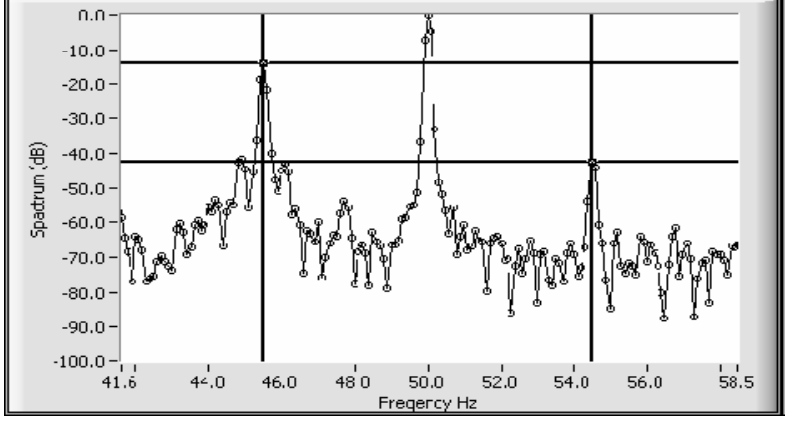

Figura 4 - Detecção de defeito no circuito rotórico através da medição do fluxo magnético: espectro de frequências do campo magnético de fugas [3]

\subsection{DIAGNÓSTICO DE AVARIAS MECÂNICAS}

\subsubsection{Defeito DE ALinhamento}

O defeito de alinhamento do rotor traduz-se no facto da folga entre o rotor e o estator ("entreferro") não ser constante ao longo de toda a periferia do rotor, originando variações da relutância do circuito magnético com a rotação do rotor e consequente formação de harmónicos na força magnetomotriz.

Daí resulta o aparecimento de frequências típicas deste fenómeno no espectro do fluxo de fugas do motor, como evidencia a Figura 5, que servem de meio de diagnóstico deste defeito [4].
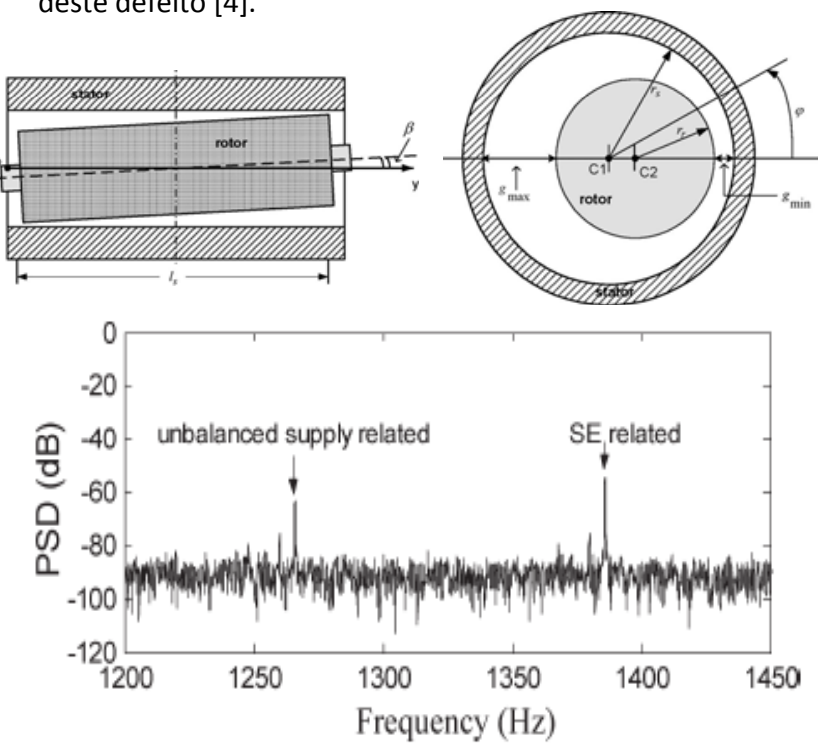

Figura 5 - Detecção de defeito de alinhamento do rotor: defeito de alinhamento longitudinal (figura esquerda em cima); desalinhamento axial (figura direita em cima); espectro de frequências da corrente de alimentação revelando o defeito de alinhamento do rotor, excentricidade estática ("SE") (figura em baixo )[4]. 


\subsubsection{DEFEITOS NOS ROLAMENTOS}

Sendo os rolamentos a causa referenciada que provoca maior taxa de avarias no motor de indução, têm-se desenvolvido diversas técnicas de detecção deste tipo de defeito recorrendo a diferentes métodos de análise espectral da corrente de alimentação e de vibrações mecânicas.

Os defeitos nos rolamentos podem ser detectados a partir da análise do espectro de frequências da corrente do motor como evidencia a Figura 6.

Também neste caso surgem novas frequências que evidenciam a existência de defeito nos rolamentos assim como o seu nível de severidade [5].
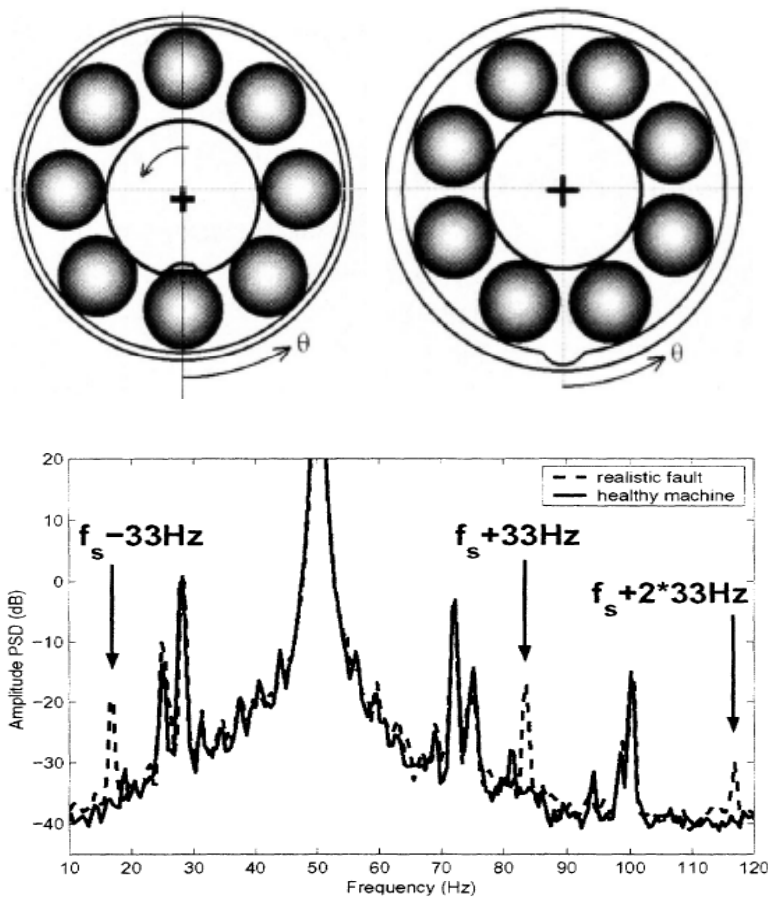

Figura 6 - Avarias nos rolamentos e sua detecção: defeito no anel interior (figura esquerda em cima); defeito no anel exterior (figura direita em cima); decomposição em frequências da corrente de alimentação do motor mostrando o espectro no caso de um motor saudável e no caso de existir um defeito nos rolamentos (figura em baixo) [5].

\subsubsection{DefEITOS NA CARGA ACCIONADA}

A Figura 7 apresenta o caso da aplicação de acelarómetros para aquisição de dados de vibração de um ventilador.

A análise das componentes espectrais de vibração e de binário (Figura 8 ) pode ser um meio complementar de apoio ao diagnóstico de um sistema electromecânico do qual se conhece a sua composição em funcionamento normal [6].

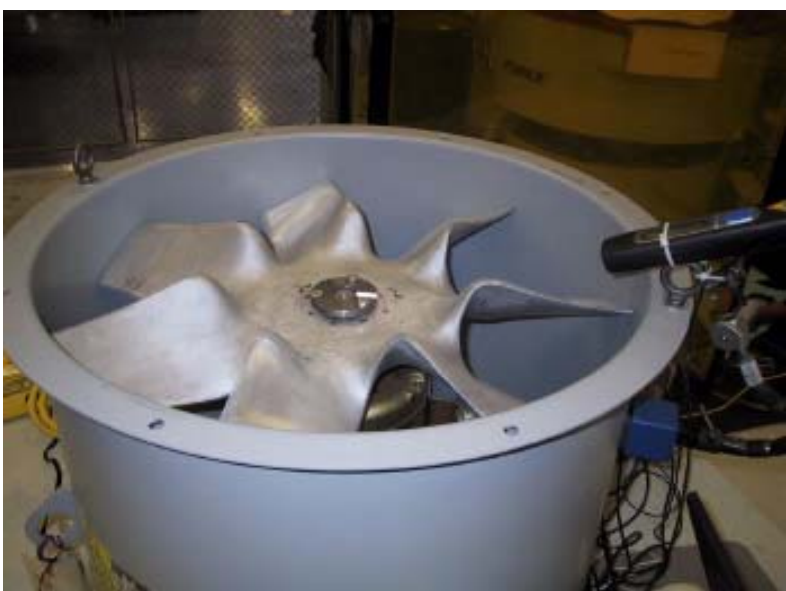

Figura 7 - Utilização de acelerómetros para aquisição de dados de vibração de um ventilador [6]

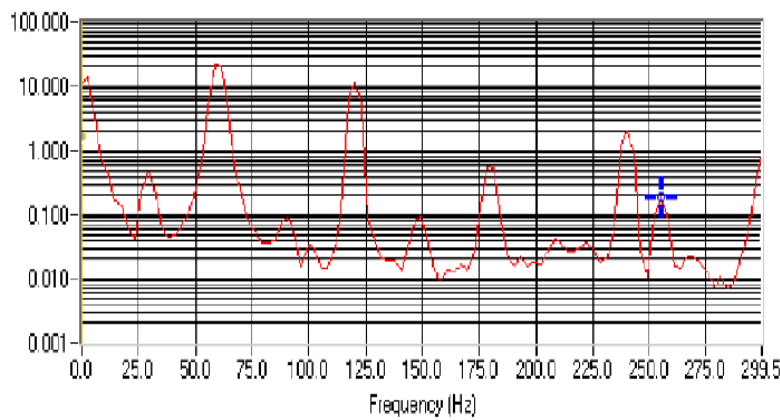

Figura 8 - Amplitude das componentes espectrais do binário ( $\mathrm{Nm}$ ) para o caso do mesmo ventilador [6]

A monitorização do valor eficaz da corrente de alimentação do motor de um elevador pode fornecer indicações úteis relativamente a variações de carga que indiciam a existência de defeitos nas partes mecânicas accionadas.

O caso apresentado na Figura 9 e 10 ilustra a detecção de um defeito existente nos dentes da roda dentada de um redutor do tipo "sem fim" [7].
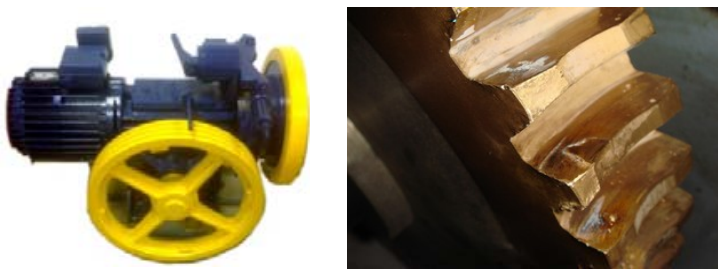

Figura 9 - Detecção de defeitos nas rodas dentadas do redutor de um elevador: motor de elevador com redutor de velocidade acoplado (esquerda); roda dentada do redutor (direita) com dente defeituoso [7] 


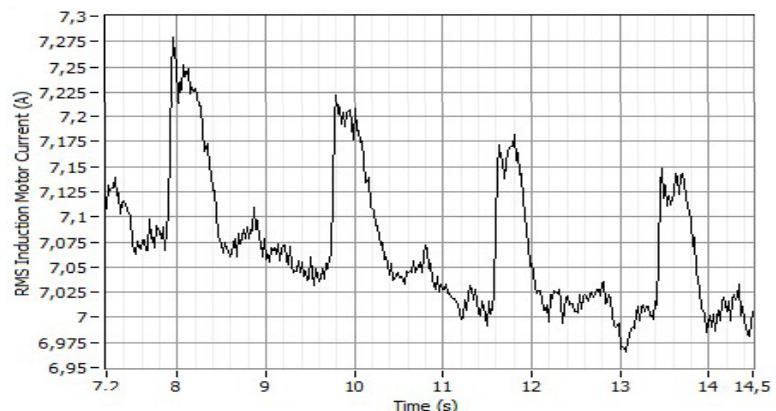

Figura 10 - Valor eficaz da corrente de alimentação do motor [7]

\section{CONCLUSÕES}

Apesar do motor de indução ser considerado uma máquina robusta e muito fiável está sujeito a diversos tipos de avarias causadas principalmente pelo envelhecimento, desgaste e fadiga mecânica dos materiais.

Dado o seu importante papel, muitos trabalhos de investigação têm sido feitos oferecendo metodologias para um diagnóstico cada vez mais eficiente.

A aplicação destas técnicas em ambiente fabril tem as suas limitações devido a diversos factores, como por exemplo, a existência de poluição harmónica proveniente de outras máquinas e a falta de registos históricos das componentes espectrais.

As ferramentas de diagnóstico apresentadas não são invasivas e podem ser aplicadas durante o funcionamento normal, contribuindo para uma manutenção preventiva eficiente através da monitorização regular do motor.

Além disso, se criteriosamente utilizadas, constituem uma mais-valia, melhorando a eficiência da equipa de manutenção e minimizando os custos de indisponibilidade e de interrupção.

Assim, para além da sua função principal de fornecer energia mecânica à carga, o motor de indução pode desempenhar um segundo papel, como transdutor eficaz e permanentemente ligado, ajudando a detectar avarias no seu interior e também na carga mecânica a ele acoplada.

\section{Bibliografia}

[1] A. J. Marques Cardoso, S. M. A. Cruz, D. S. B. Fonseca, "Inter-turn stator winding fault diagnosis in three-phase induction motors, by Park's vector approach", IEEE Transactions on Energy Conversion, vol. 14, pp. 595-598, 1999.

[2] G. G. Acosta, C. J. Verucchi, E. R. Gelso, "A current monitoring system for diagnosing electrical failures in induction motors", Mechanical Systems and Signal Processing, vol. 20, pp. 953-965, 2006.

[3] A. Yazidi, H. Henao, G. A. Capolino, "Broken rotor bars fault detection in squirrel cage induction machines", IEEE International Conference on Electric Machines and Drives, 2005, pp. 741-747.

[4] L. Xiaodong, W. Qing, S. Nandi, "Performance analysis of a three-phase induction machine with inclined static eccentricity", IEEE Transactions on Industry Applications, vol. 43, pp. 531-541, 2007.

[5] M. Blodt, P. Granjon, B. Raison, G. Rostaing, "Models for bearing damage detection in induction motors using stator current monitoring," IEEE International Symposium on Industrial Electronics, 2004, pp. 383-388 vol. 1.

[6] E. Wiedenbrug, D. Doan, "Comparison of duct-mounted vibration and instantaneous airgap torque signals for predictive maintenance of vane axial fans", International Conference on Measurement and Control, 2004, pp. 209213.

[7] A. Q. Flores, A. J. M. Cardoso, J. B. Carvalho, "The induction motor as a mechanical fault sensor in elevator systems " apresentado na conferência "11CHLIE", 11a . Conferencia Hispano-Lusa de Ingeniería Eléctrica, Saragoça, Espana, 2009. 